\title{
BMJ Open Non-pharmacological self-management for people living with migraine or tension-type headache: a systematic review including analysis of intervention components
}

\author{
Katrin Probyn, ${ }^{1}$ Hannah Bowers, ${ }^{1}$ Dipesh Mistry, ${ }^{2}$ Fiona Caldwell, ${ }^{1}$ \\ Martin Underwood, ${ }^{2}$ Shilpa Patel, ${ }^{2}$ Harbinder Kaur Sandhu, ${ }^{2}$ Manjit Matharu, ${ }^{3}$ \\ Tamar Pincus, ${ }^{1}$ On behalf of the CHESS team.
}

To cite: Probyn $\mathrm{K}$,

Bowers H, Mistry D, et al. Non-pharmacological selfmanagement for people living with migraine or tension-type headache: a systematic review including analysis of intervention components. BMJ Open 2017;7:e016670. doi:10.1136/ bmjopen-2017-016670

- Prepublication history and additional material for this paper are available online. To view these files please visit the journal online (http://dx.doi. org/10.1136/bmjopen-2017016670).

Received 6 March 2017 Revised 7 June 2017 Accepted 21 June 2017

CrossMark

${ }^{1}$ Department of Psychology, Royal Holloway University of London, Egham, Surrey, UK ${ }^{2}$ Clinical Trials Unit, Warwick Medical School, Warwick University, Coventry, UK ${ }^{3}$ Headache Group, Institute of Neurology and The National Hospital for Neurology and Neurosurgery, London, UK

Correspondence to Mrs. Katrin Probyn; katrin.probyn@rhul.ac.uk

\section{ABSTRACT}

Objectives To assess the effect of non-pharmacological self-management interventions against usual care, and to explore different components and delivery methods within those interventions

Participants People living with migraine and/or tensiontype headache

Interventions Non-pharmacological educational or psychological self-management interventions; excluding biofeedback and physical therapy. We assessed the overall effectiveness against usual care on headache frequency, pain intensity, mood, headache-related disability, quality of life and medication consumption in meta-analysis. We also provide preliminary evidence on the effectiveness of intervention components and delivery methods.

Results We found a small overall effect for the superiority of self-management interventions over usual care, with a standardised mean difference (SMD) of $-0.36(-0.45$ to -0.26$)$ for pain intensity; $-0.32(-0.42$ to -0.22$)$ for headache-related disability, $0.32(0.20$ to 0.45$)$ for quality of life and a moderate effect on mood (SMD $=0.53(-0.66$ to -0.40$)$ ). We did not find an effect on headache frequency $(\mathrm{SMD}=-0.07(-0.22$ to 0.08$))$. Assessment of components and characteristics suggests a larger effect on pain intensity in interventions that included explicit educational components $(-0.51(-0.68$ to -0.34$)$ vs $-0.28(-0.40$ to $-0.16))$; mindfulness components $(-0.50(-0.82$ to -0.18$)$ vs $0.34(-0.44$ to -0.24$)$ ) and in interventions delivered in groups vs one-to-one delivery $(0.56(-0.72$ to -0.40$)$ vs $-0.39(-0.52$ to -0.27$))$ and larger effects on mood in interventions including a cognitive-behavioural therapy (CBT) component with an SMD of $-0.72(-0.93$ to -0.51$)$ compared with those without CBT $-0.41(-0.58$ to -0.24$)$. Conclusion Overall we found that self-management interventions for migraine and tension-type headache are more effective than usual care in reducing pain intensity, mood and headache-related disability, but have no effect on headache frequency. Preliminary findings also suggest that including CBT, mindfulness and educational components in interventions, and delivery in groups may increase effectiveness.

\section{Strengths and limitations of this study}

- We prospectively registered this review with PROSPERO and followed the PRISMA (Preferred Reporting Items for Systematic Reviews and MetaAnalyses) guidelines for reporting in systematic reviews.

- We quantified effects of self-management interventions against usual care in meta-analysis.

- We identified possible components and characteristics associated with better outcomes in qualitative comparison of studies' effect sizes.

- Long-term effectiveness of interventions could not be assessed.

- We identified large heterogeneity among studies, interventions; outcome measures used and followup times.

Trial registration number PROSPERO

2016:CRD42016041291

\section{INTRODUCTION}

Non-pharmacological self-management interventions have been promoted as a promising approach for helping people with intractable chronic conditions. ${ }^{1}$ For migraine and tensiontype headaches self-management can be used either alongside pharmacological interventions or as a stand-alone therapy, particularly when other treatment options have failed or a person prefers a non-pharmacological intervention. ${ }^{2}$ The underlying models conceptualise the headache management as strongly influenced by behavioural factors. ${ }^{3}$ Through consideration of factors influencing the headache, these treatments are aimed at enabling patients to handle pain and symptoms associated with their headaches more effectively. ${ }^{4}$ 
The rationale for promoting self-management interventions is grounded in the hypothesis that people can learn ways to help themselves manage their headaches better and that this can have a positive effect on both physical symptoms and functional capacity. ${ }^{2}$ Typically such interventions include a variety of components, either with or without conjunctive pharmacological therapy. Two common approaches, including biofeedback and exercise/physical therapy, have been reviewed comprehensively elsewhere and are not included in the present review. ${ }^{5-11}$ The current literature provides preliminary evidence to support the use of self-management interventions overall, featuring various different approaches but interventions differ considerably in terms of content and delivery.

The only non-pharmacological treatment recommended in National Institute and Health Care Excellence (NICE) guidelines is a course of acupuncture for tension-type headache. ${ }^{12}$ In contrast, self-management interventions have an established place in the management of a range of chronic diseases and are successfully used in other chronic pain conditions. ${ }^{13-15}$ We therefore set out to quantify the effect of self-management interventions, which are commonly included in trials in migraine and tension-type headache populations, and to explore the effects of interventions that included specific components compared with those that did not include them. This study was carried out to inform the development of a complex non-pharmacological intervention for the Chronic Headache Education and Self-management Study (CHESS). As the NICE guidelines specifically call for pragmatic randomised controlled trials (RCTs) of psychological interventions, CHESS aims to develop a sustainable non-pharmacological educational self-management intervention for people with chronic migraine/ and or tension-type headache. In this review, we therefore focus on educational and psychological approaches and sustainable interventions without the use of any apparatus and exclude studies including biofeedback or exercise/ physical therapy. Because we aimed to pool data, we focus exclusively on eligible interventions against treatment as usual or waiting list controls.

Specifically, our aims in this review were to provide an overall effect size of non-pharmacological self-management interventions against usual care, and to explore different components and delivery methods within those interventions.

\section{METHODS}

We prospectively registered this review with the International Prospective Register of Systematic Reviews ${ }^{16}$; CRD42016041291, ${ }^{16}$ and we followed the PRISMA (Preferred Reporting Items for Systematic Reviews and Meta-Analyses) guidelines for reporting in systematic reviews. ${ }^{17}$

\section{Identification of studies}

We searched for peer-reviewed publications in the Cochrane library, Medline, Embase, PsycINFO and Web of Science. As the definition of self-management interventions in general has changed substantially over the last three decades, we limited the search to January 1980 to June 2016. We based our search strategies on MeSH indexing terms and free text terms. The MeSH headache terms used were based on NICE guidelines recommendations. ${ }^{12}$ Our search targeted two headache conditions (migraine and tension-type headache), but also included other headache conditions to avoid missing trials with mixed groups (full search strategies can be found in online supplementary data).

Search words included: episodic cluster headache or cluster headache or episodic tension headache or headache or chronic tension headache or new daily persistent headache or secondary headache or tension headache or chronic daily headache/ or chronic cluster headache or primary headache, migraine aura or migraine or migraine with aura or migraine without aura combined with psychosocial disorder, psychosocial rehabilitation, mindfulness, cognitive therapy or CBT, group therapy, self-management or self-management or self-care, training programme, behavioural or behavioural, pain treatment. We used alternative spellings and truncations as appropriate. We supplemented our search with backward citation tracking. Only studies published in English were included. We excluded any grey literature, dissertations and conference proceedings.

The search results were managed using EPPI reviewer 4 software. $^{18}$ We initially screened the records by title and abstracts against inclusion/exclusion criteria, this was shared between two reviewers with $10 \%$ of records checked for concordance; any disagreement was resolved through discussion. Articles for possible inclusion were retrieved in full and assessed for inclusion/exclusion by two reviewers independently.

\section{Study design}

We included peer-reviewed RCTs with one or more relevant self-management interventions compared with usual care. For the purposes of this review (data pooling), we excluded study designs comparing the effects of two or more treatments to each other. We excluded any non or pseudorandomised studies, and interventions that are adjunctive to pharmacological treatment.

\section{Study population}

We only included adults ( $\geq 18$ years old) with relevant headache diagnoses. We excluded studies with a mixture of chronic pain conditions (other than headache conditions) and any paediatric studies. We did not limit for headache frequency and included trials in both chronic and episodic groups.

\section{Interventions}

We included non-pharmacological educational and/or psychological self-management interventions targeting the individual's headache-related behaviours and physiological response without the use of any apparatus. For 
'self-management' we used the definition established in a previous systematic review of self-management interventions for musculoskeletal pain ${ }^{19}$ as 'a structured, taught, or self-taught course or intervention programme principally aimed at patients (rather than carers or lay advisors) with the goal of improving the participants' health status or quality of life by teaching them skills to apply to everyday situations.'

We excluded interventions utilising physical therapy and exercise or biofeedback interventions, which are reviewed elsewhere..$^{5-7} 1011$ We also excluded interventions assessing pharmacological interventions or assessing self-management in conjunction with pharmacological interventions.

We extracted content of each intervention. We then reviewed the extracted components and developed an overarching framework resulting in four main categories. The framework was developed through a consensus within the research team. The categories included:

- A cognitive-behavioural component aimed at changing headache-related behaviours and beliefs (including cognitive-behavioural therapy/stress management therapy, trigger management training).

- Patient education that aimed to increase participants' skills and knowledge and to enable participants to deploy these enhanced skills in aspects of their lives beyond the intervention.

- Mindfulness-based and acceptance-based techniques involving training participants to engage in self-regulation of attention through increasing awareness of, and accepting, present thoughts, feelings and physical sensations.

- Relaxation training that aims to increase the patients' control over physiological responses to their headache, lower sympathetic arousal and reduce stress and anxiety.

\section{Comparisons}

We included studies that compared their interventions to usual care or to a waiting list control within the trial (which is equivalent to usual care). We only included studies with this type of control group as described by the authors as 'usual care', 'care as usual', 'standard medical care' or 'waiting list control'. We excluded any studies using any other types of control groups to ensure sufficient similarity across studies to pool data.

\section{Outcome measures}

We included studies using validated outcome assessment tools for at least one of the following headache-related outcome measures: headache frequency (headache days per month), pain intensity, headache-related disability, measures of quality of life, mood (measures of anxiety and depression) or medication consumption.

\section{Quality assessment}

Two reviewers independently assessed the risk of bias in included RCTs by examining randomisation method, allocation concealment, attrition, masked outcome assessment and intention-to-treat analyses. This approach was adapted from the Cochrane Handbook. ${ }^{20}$ Disagreement was resolved through discussion.

\section{Data extraction}

For each included study, we extracted published information on details of delivered interventions and the included components, including the authors' description and labelling of these. When all components were extracted, we developed a categorisation system, and grouped the components, according to the primary mechanism they targeted, as described above.

We extracted information on country, population, sample size, number of intervention arms, control intervention, description of intervention content, delivery mode (classified as group, individual, mixed or remote; ie, internet, mail, telephone), intervention provider (psychologist/therapist or nurse/allied health professional or no contact, though mail instructions, DVDs, web-based instructions), use of any additional delivery modes (homework, email/telephone support), control intervention, outcome measures, follow-up times for trial outcomes and duration of the intervention in total hours.

We extracted final value data for the intervention arm and the control arm for each of our included outcome categories (where assessed and reported in the paper). We extracted final values at baseline and post-treatment follow-ups (effect size, CI and N) for treatment and control groups.

\section{Data analysis}

We standardised the measure-specific values across outcome categories headache frequency, pain intensity, mood. We combined measures on anxiety and depression into a single 'mood' category because of emerging theoretical and evident conceptualising recognising the substantial overlap between the two, for example, the recommendation to use a single combined measure labelled distress ${ }^{21}$, headache-related disability, quality of life and medication consumption.

Where some studies had more than one type of self-management intervention arm, we included both arms in the meta-analyses. This meant that there was some double counting for the sample size in the control arms. This inclusive approach can result in unit of analysis of errors, ${ }^{20}$ so we tested the impact of including the multiple study arms by performing a sensitivity analysis excluding these studies. We also conducted sensitivity analyses for high-quality and low-quality RCTs. We only included one outcome measure per study for each outcome category to avoid unit of analysis issues.

\section{Overall effectiveness meta-analysis}

We produced the overall pooled effect size for each outcome across studies by combining the final value data in the intervention and control arm for each study and calculating 
standardised mean differences (SMD) using Stata V.14.1. ${ }^{22}$ We present SMD values for effect sizes (with 95\% CIs).

\section{Intervention component analysis}

We compared the effects of the presence or absence of different intervention components and delivery modes on outcomes by comparing the pooled SMDs of studies with versus studies without certain intervention components and characteristics.

For these comparisons we limited our analyses to comparisons where we had outcome data available from at least 10 studies overall per comparison.

For all analyses the pooled SMDs were interpreted using Cohen's $d$ proposal in which an effect size of $<0.2$ is considered minor, $\geq 0.2-0.5$ is considered small, $\geq 0.5-0.8$ is considered moderate and $\geq 0.8$ is considered large. ${ }^{23}$ To assess heterogeneity or variability between studies, $\mathrm{I}^{2}$ statistics were interpreted following the recommendations in the Cochrane Handbook ${ }^{20}$ in which $\mathrm{I}^{2} \geq 50 \%$, with a statistically significant $p$ value of $<0.05$ for the w2 test, indicates substantial to considerable heterogeneity.

Our analysis was limited to post-treatment follow-up only. Longer term follow-up was only reported for a very limited number of studies and at different follow-up times, hence we could not analyse this. We could not assess the effect of contact time/length of intervention in this analysis due to insufficient information from primary studies.
Assessment of potential publication bias

We generated a funnel plot (scatter diagram) of SMDs against the SE for the SMD to illustrate data distribution and explore potential publication bias.

\section{RESULTS}

\section{Selected studies}

We identified $16 \mathrm{RCTs}^{24-39}$ testing 21 non-pharmacological self-management interventions that fit our eligibility criteria compared with usual care (see figure 1 and table 1).

All 16 included studies reported final value data (no change scores) for their outcome measures; six were from the USA, three from Iran, two each from the Netherlands and Australia, and one each from Germany, the UK and Sweden. Of these studies, four each were specifically for migraine or tension-type headache only and the remaining eight were for mixed conditions of migraine and tension-type headache.

\section{Quality assessment}

Seven of the 16 studies were of higher quality (high or medium) than the rest. We performed a sensitivity analysis comparing higher quality to lower quality studies for all of our performed analyses. This analysis did not find a difference in overall effect size in the higher quality

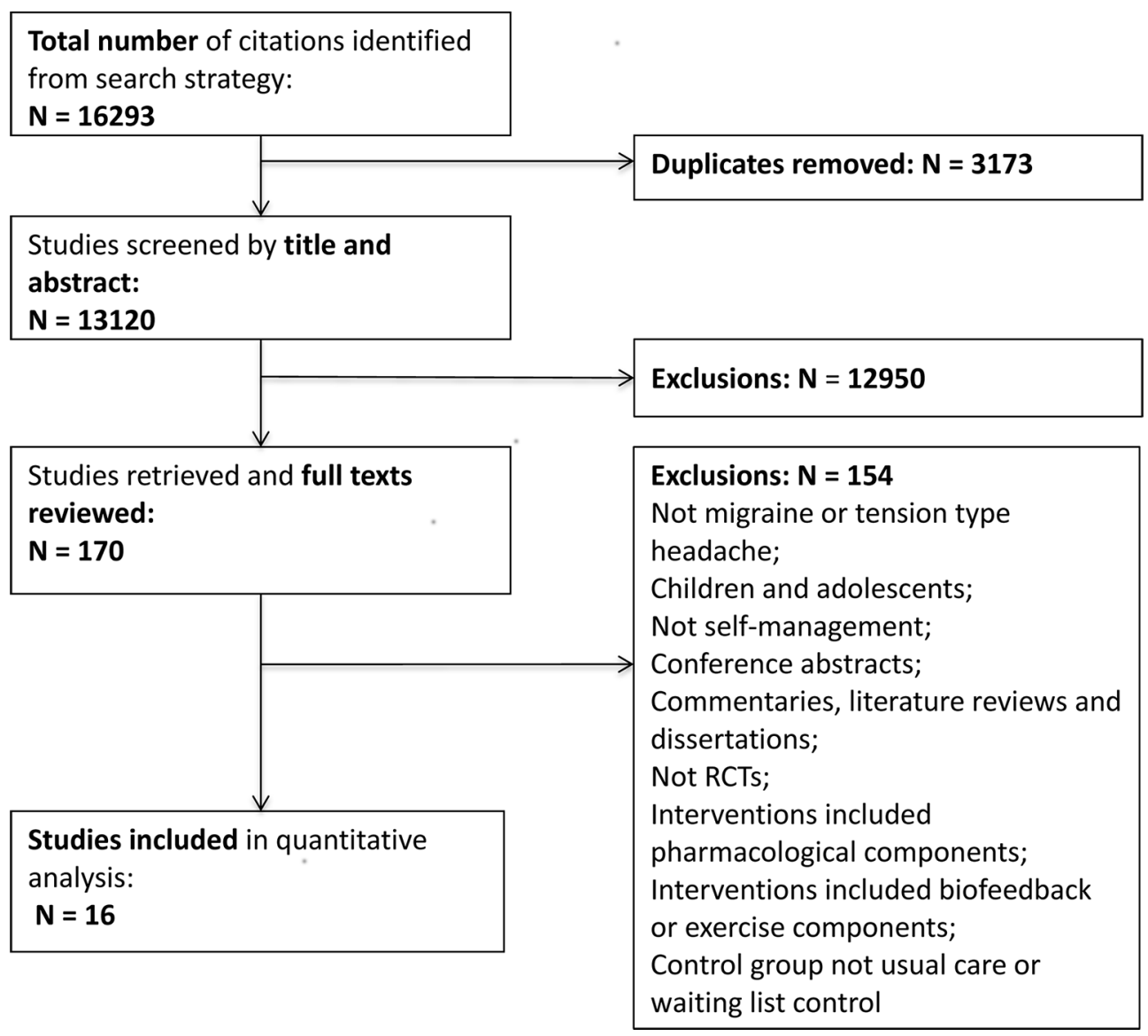

Figure 1 Study flow chart. RCT, randomised controlled trial. 


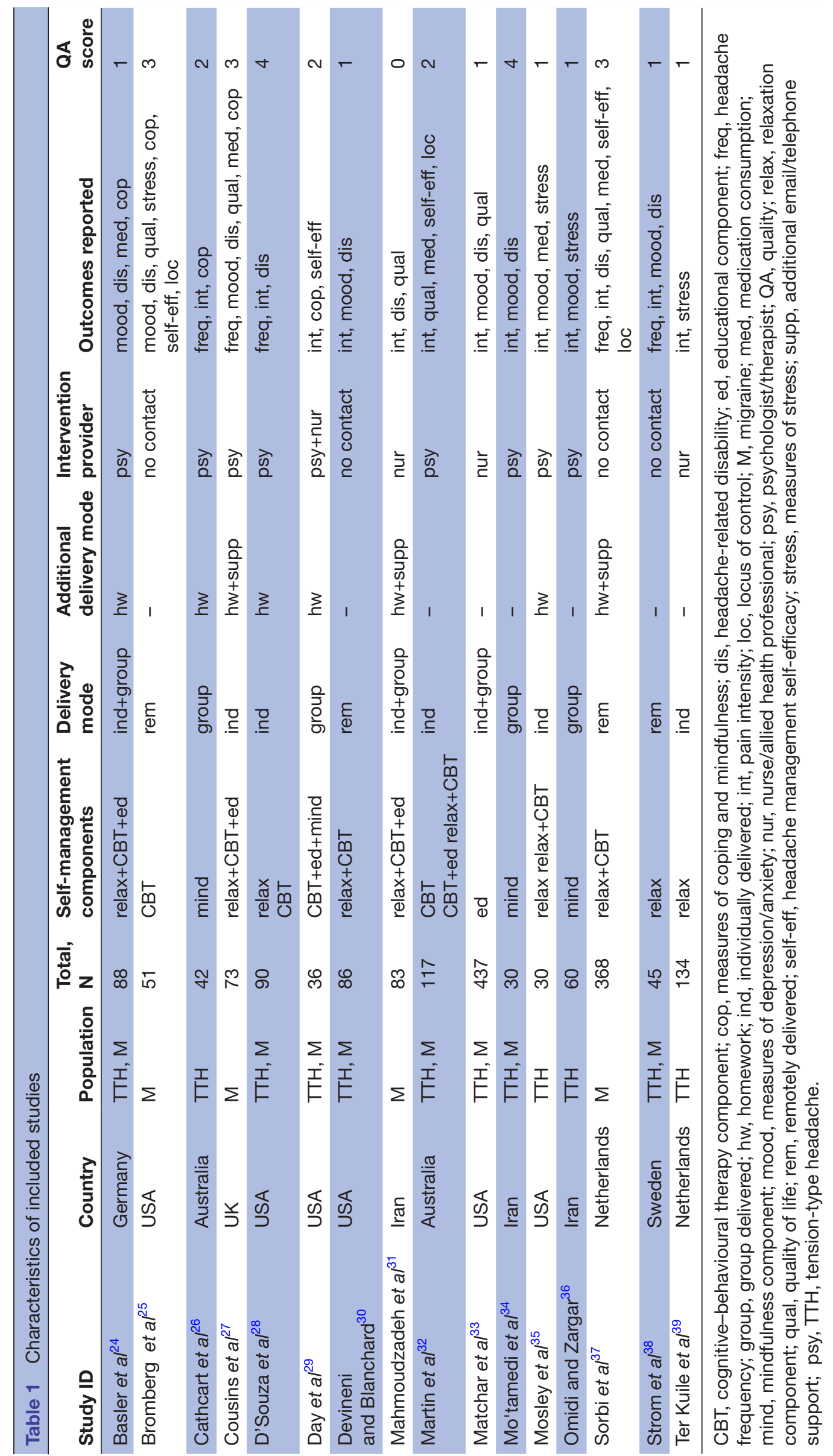


studies when compared with the lower quality studies. For quality of studies see table 2 .

\section{Overall effectiveness}

None of our analyses showed the control group to be superior to the intervention group. The overall effectiveness of included self-management interventions was greater than control with a moderate effect size for mood (depression and anxiety), a small effect size for the outcomes pain intensity, headache-related disability and quality of life; and a minor effect size for medication consumption. There were no statistically significant additional improvements on the outcome headache frequency (see table 3 and online Supplementary files 1-6).

\section{Comparison of effect sizes of components and characteristics}

We assessed effect sizes of components and delivery characteristics where we had useable outcome data from a minimum of at least 10 studies overall.

We present SMD values for effect sizes (with 95\% CIs) for all of those comparisons (see tables 4 and 5) and results are described below for each of our selected areas of interest for intervention components and delivery characteristics.

We did not have sufficient data to assess the effectiveness of components on all outcomes. Hence, there are many outcomes and comparisons we have not been able to analyse.

\section{Effect sizes of self-management interventions including a cognitive-behavioural therapy component}

Ten of the included studies included a cognitivebehavioural therapy (CBT) component in 13 intervention arms. In three intervention arms, a CBT intervention was used on its own, whereas in the majority of 10 studies, it was used alongside other components, and the remaining six studies did not include CBT. Our analysis shows that including a CBT component doubles the effect size on mood (anxiety and depression). Including CBT in the intervention did not make a difference on headache-related disability or pain intensity (see table 4 ).

\section{Effect size of self-management interventions including an} educational component

Five studies included an explicit educational component in their intervention; four studies used this component alongside other components and one study used only education in their intervention, the remaining 11 studies did not use this component in their intervention. The effect sizes for interventions that included an explicit educational component are nearly double the effect of those that did not include education on the outcomes pain intensity and headache-related disability (see table 4).

\section{Effect size of self-management interventions including a} mindfulness component

Four studies used a mindfulness-based approach in their intervention (three alongside other component, one study used it individually); 12 did not use this component. Including mindfulness components is associated with a larger effect size on pain intensity (see table 4 ).

Effect size of self-management interventions including a relaxation component

Nine intervention arms included relaxation components, whereas the remainder $(n=7)$ did not. Including a relaxation component did not improve the effectiveness on pain intensity or headache-related disability (see table 4 ).

\section{Effect sizes for delivery mode}

Four of the studies involved group interventions; five individually delivered interventions, three a mixture of both, whereas the remaining four studies were delivered remotely (see table 5). Interventions delivered to groups had a larger effect than interventions delivered individually. There appeared to be little difference in effect size between interventions that were delivered face-to-face and those that were delivered remotely (see table 5). We could not analyse the effect of interventions that were a mixture of individual and group delivery. Five studies included homework and three studies included homework and additional email/telephone support in their intervention, whereas the remainder of studies did not. There did not appear to be a difference between the interventions with these added features and those without them (see table 5).

\section{Effect sizes for intervention provider}

Half of the interventions ( 8 of 16) were delivered by a clinician with a qualification in the practice of psychology (psychologist or a psychotherapist), three were delivered by a nurse or allied health professional, one by a combination of the two and the remaining four study interventions were delivered with no contact (by mail, email, DVD instructions or web-based) (see table 5). There was no evidence that interventions delivered by a psychologist or psychotherapists were more, or less, effective than interventions delivered by a nurse or allied health professional (see table 5).

\section{Studies with more than one self-management arm}

Four studies had more than one intervention arm and we included all arms in the meta-analyses. ${ }^{28} 323539$ Our sensitivity analysis showed that the removal of these studies did not alter our conclusions.

\section{Heterogeneity and publication bias}

Overall substantial heterogeneity of variability between results was shown with most comparisons having an $\mathrm{I}^{2}$ of $>50 \%$. The funnel plot symmetry suggested publication bias was unlikely and that no further exploration was needed to explain the distribution of our SMDs. The bases of the plots are not skewed to one side. The effect of the smaller studies, with larger SEs, that have more scatter of the magnitude of effect (ie, being less precise) does not cause the funnel plot to be asymmetric/skewed 


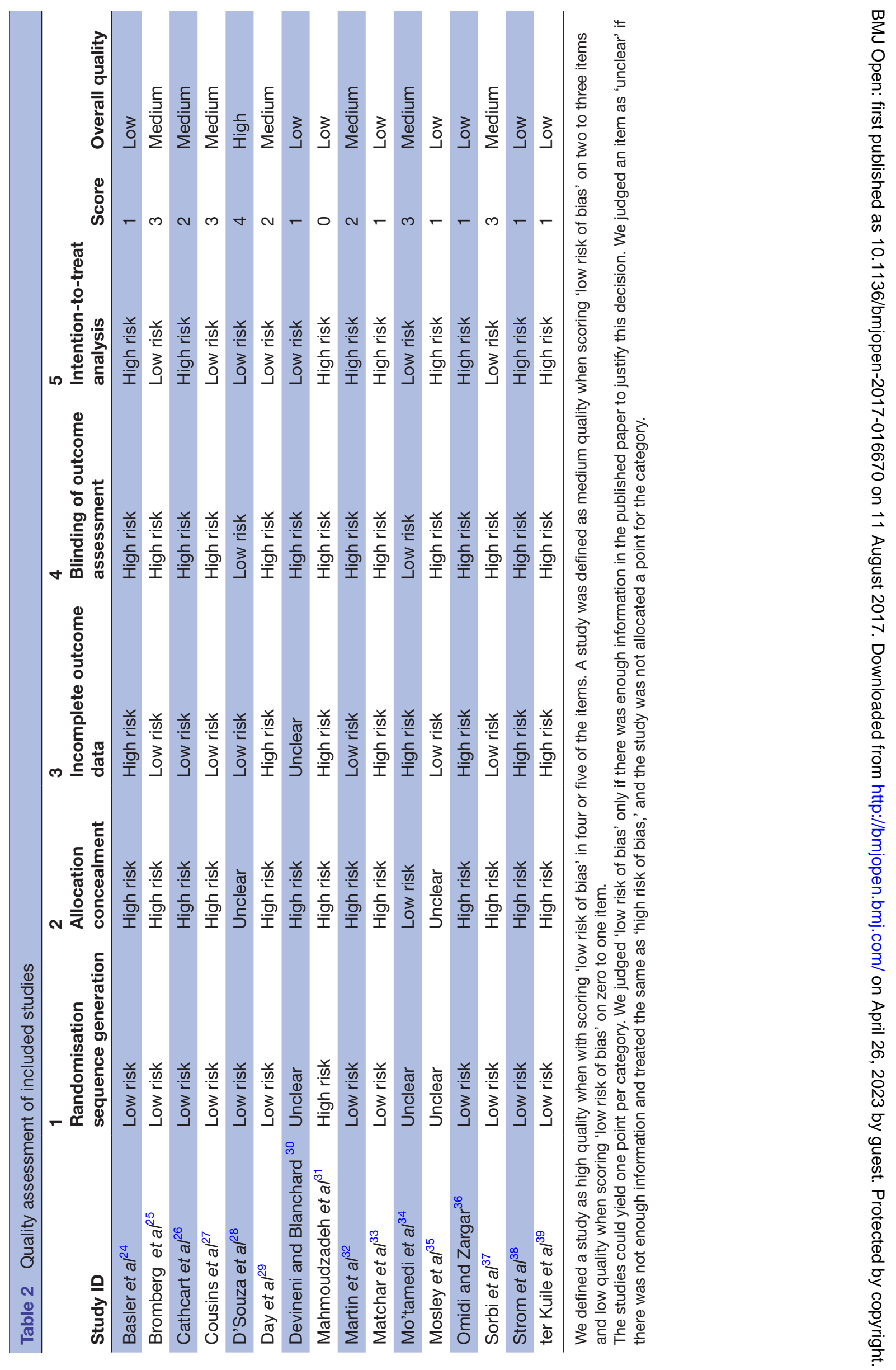


Table 3 Overall effectiveness of self-management versus usual care

\begin{tabular}{lll}
\hline & $\begin{array}{l}\text { Treatment versus } \\
\text { control } \\
\text { SMD }(\mathbf{C l})\end{array}$ & $\begin{array}{l}\text { N } \\
\text { participants } \\
\text { (studies) }\end{array}$ \\
\hline Headache frequency & $-0.07(-0.22$ to 0.08$)$ & $717(6)$ \\
\hline $\begin{array}{l}\text { Pain intensity } \\
\text { Mood }\end{array}$ & $-0.36(-0.45$ to -0.26$)$ & $1749(18)$ \\
$\begin{array}{l}\text { Headache-related } \\
\text { disability }\end{array}$ & $-0.53(-0.66$ to -0.40$)$ & $987(10)$ \\
$\begin{array}{l}\text { Quality of life } \\
\begin{array}{l}\text { Medication } \\
\text { consumption }\end{array}\end{array}$ & $-0.32(0.20$ to 0.45$)$ & $1116(5)$ \\
\hline
\end{tabular}

Direction of effect: for outcomes frequency, pain intensity, mood, headache-related disability and medication consumption, negative values are beneficial; for the outcome quality of life a positive value is beneficial.

*Including double-counted control arms in studies with multiple treatment arms.

SMD, standardised mean differences.

to one side, hence we conclude publication bias to be unlikely (online supplementary files 7-12).

\section{DISCUSSION}

\section{Findings and implications}

We identified 16 studies that compared 21 non-pharmacological self-management programmes that fit our eligibility criteria to treatment as usual for people with migraine and/or tension-type headache. Overall non-pharmacological self-management appears to be slightly more effective in improving pain intensity
$(\mathrm{SMD}=-0.36(-0.45$ to -0.26$))$, headache-related disability $(\mathrm{SMD}=-0.32(-0.42$ to -0.22$))$, quality of life $(\mathrm{SMD}=0.32$ $(0.20$ to 0.45$))$ and medication consumption $(\mathrm{SMD}=-0.18$ $(-0.33$ to -0.03$))$, and moderately more effective than usual care in improving mood (anxiety and depression) $(\mathrm{SMD}=-0.53(-0.66$ to -0.40$))$. Assessed self-management interventions did not improve measures on headache frequency $(\mathrm{SMD}=-0.07(-0.22$ to 0.08$))$.

Our findings are promising preliminary evidence, but we advise that it should be treated with caution because of the large heterogeneity within interventions and population and small number of included studies. Other reviews that have assessed different self-management approaches for migraine and or tension-type headache also found substantial heterogeneity in included studies. ${ }^{10} 1140$ We note that the inclusion criteria and interventions investigated in these reviews vary and are partially different from ours, but interventions and studies included partially overlap and together the reviews indicate that there is a clear need for definitive studies in the area. We propose that future trials consider to identify mechanisms by single intervention studies, and that reviewers analyse full sets of data rather than mean values.

We identified components and characteristics of interventions in a series of meta-analyses that allowed for some comparison between effect sizes in interventions that included specific components (CBT, education, mindfulness and relaxation) and delivery characteristics, and interventions that did not include them.

We found evidence to suggest that delivery of interventions in groups may be more effective than individual delivery, but were unable to test a mixture of group and individual delivery because of paucity of such trials. This

Table 4 Comparing the presence and absence of components within interventions

\begin{tabular}{|c|c|c|c|c|}
\hline \multicolumn{2}{|c|}{ Intervention components } & \multirow{2}{*}{$\begin{array}{l}\text { Intensity SMD (Cl) } \\
\text { N participants (studies) } \\
-0.36(-0.49 \text { to }-0.22) \\
950(8)\end{array}$} & \multirow[t]{2}{*}{$\begin{array}{l}\text { Mood SMD (Cl) } \\
\text { N participants (studies) }\end{array}$} & \multirow{2}{*}{$\begin{array}{l}\text { Headache-related } \\
\text { disability SMD (Cl) } \\
\text { N participants (studies) } \\
-0.29(-0.43 \text { to }-0.15) \\
822(7)\end{array}$} \\
\hline Relaxation & With & & & \\
\hline & Without & $\begin{array}{l}-0.36(-0.50 \text { to }-0.21) \\
799(7)\end{array}$ & & $\begin{array}{l}-0.36(-0.51 \text { to }-0.21) \\
718(4)\end{array}$ \\
\hline \multirow[t]{2}{*}{ CBT } & With & $\begin{array}{l}-0.38(-0.52 \text { to }-0.24) \\
836(7)\end{array}$ & $\begin{array}{l}-0.72(-0.93,-0.51) \\
405(5)\end{array}$ & $\begin{array}{l}-0.36(-0.49 \text { to }-0.23) \\
949(7)\end{array}$ \\
\hline & Without & $\begin{array}{l}-0.34(-0.47 \text { to }-0.20) \\
913(8)\end{array}$ & $\begin{array}{l}-0.41(-0.58,-0.24) \\
582(5)\end{array}$ & $\begin{array}{l}-0.26(-0.43 \text { to }-0.10) \\
591(4)\end{array}$ \\
\hline \multirow[t]{2}{*}{ Education } & With & $\begin{array}{l}-0.51(-0.68 \text { to }-0.34) \\
605(4)\end{array}$ & & $\begin{array}{l}-0.42(-0.58 \text { to }-0.27) \\
681(4)\end{array}$ \\
\hline & Without & $\begin{array}{l}-0.28(-0.40 \text { to }-0.16) \\
1144(10)\end{array}$ & & $\begin{array}{l}-0.24(-0.38 \text { to }-0.11) \\
859(6)\end{array}$ \\
\hline \multirow[t]{2}{*}{ Mindfulness } & With & $\begin{array}{l}-0.50(-0.82 \text { to }-0.18) \\
168(4)\end{array}$ & & - \\
\hline & Without & $\begin{array}{l}-0.34(-0.44 \text { to }-0.24) \\
1581(9)\end{array}$ & & - \\
\hline
\end{tabular}

$\mathrm{CBT}$, cognitive-behavioural therapy; SMD, standardised mean differences. 
Table 5 Assessing the effect of intervention characteristics

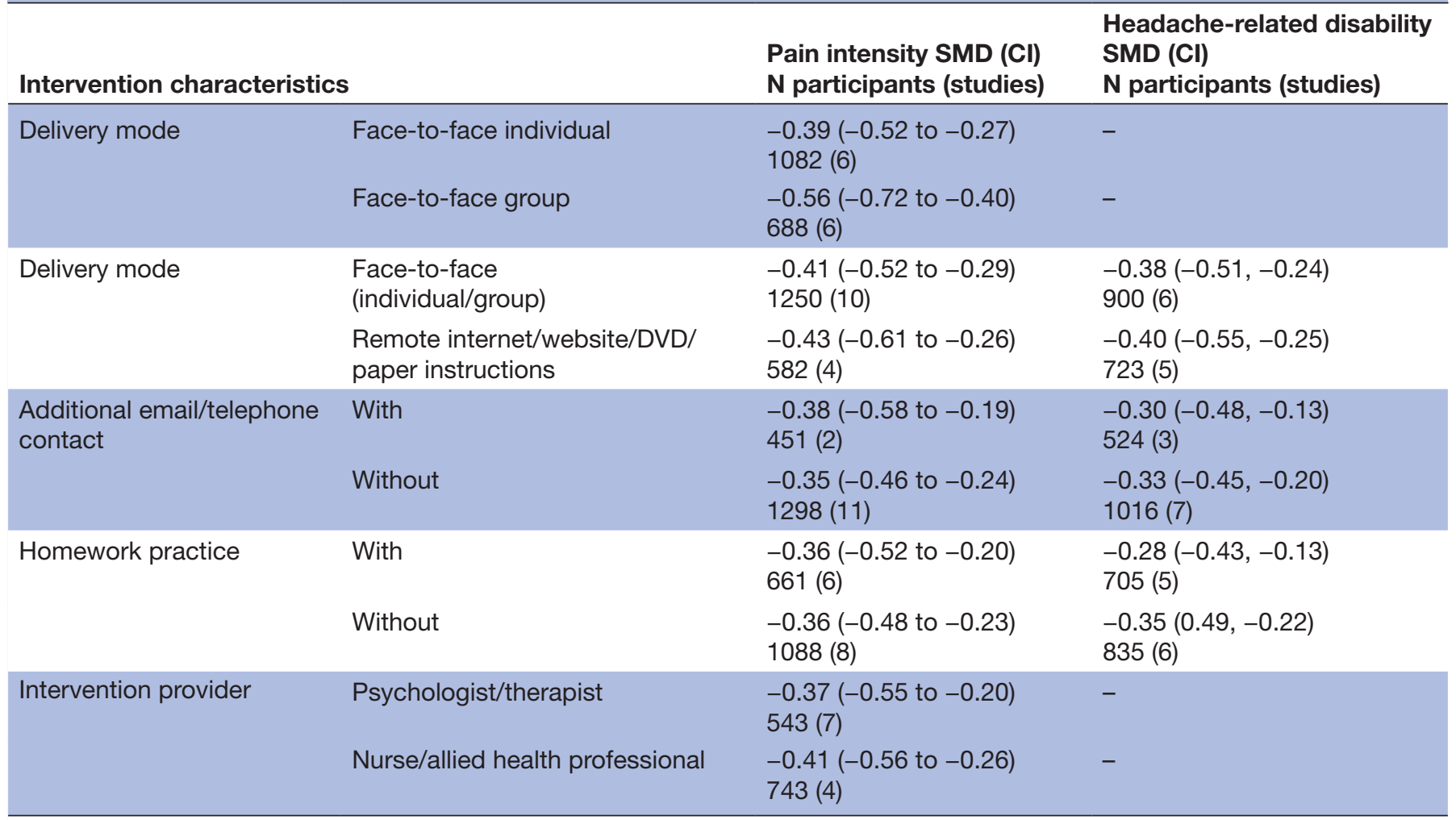

SMD, standardised mean differences.

is in line with a recent review of self-management for chronic musculoskeletal pain ${ }^{19}$ and our findings support the hypothesis from previous research that group delivery may build confidence, increase social interaction and promote integration into society. ${ }^{19}$

We also found support, in this review, for the inclusion of cognitive-behavioural components. In other groups with chronic pain, psychological interventions generally have been demonstrated to produce moderate effects on physical function and quality of life. ${ }^{13}$ Two recently published reviews ${ }^{10} 11$ assessing psychological interventions for migraine concluding that such interventions can be effective, however advise that the current state of evidence does not allow to draw conclusions because of heterogeneity among trials.

Our review also supports the use of mindfulness-based and acceptance-based approaches. Although the current evidence base in the headache literature is limited, acceptance-based therapies are seen as promising treatment approaches. ${ }^{2}$ According to Smitherman et $a l$ acceptance-based therapies are gaining popularity, mirroring a larger trend in chronic pain treatment in general. ${ }^{2}$ In other related fields mindfulness-based techniques or mindfulness-based cognitive therapy is more implemented and successfully used, ${ }^{41} 42$ though more headache-specific research is needed to recommend the use of these approaches.

We found evidence for the inclusion of explicit educational components in interventions. This fits well with current evidence suggesting that the most reassuring aspect of consultations is the delivery of information, in the form of explanations ${ }^{43}$ and maps onto the findings from a recent review assessing therapeutic patient education for migraine and concluding moderate effectiveness of interventions involving an explicit patient education element. $^{40}$

There was no difference between the delivery from professionals qualified in psychology and those who were not, which is an important preliminary finding as it could have considerable cost implications. Interventions delivered by a nurse/allied health professional may prove cost-effective and increase access to the intervention, which will need to be assessed in further research. However, we could not explore specific qualifications and competencies of professionals delivering the interventions, as such detail was not provided in primary papers.

We were unable to explore intervention duration and contact hours, which were insufficiently and inconsistently reported to quantify the effect of shorter interventions in comparison to longer ones. Previous research has reported that attendance can be an issue in self-management interventions if they are lengthy ${ }^{44}$ and therefore suggest shorter interventions with fewer contact hours to minimise that potential problem. Cost-effectiveness of minimal contact or shorter interventions is also suggested in the literature. ${ }^{45}$

Categorising outcomes was difficult, as measures used in primary trials varied considerably. The recommended primary measures to use in behavioural headache research are headache frequency/headache days per month, 
alongside the additional use of secondary measures including different pain intensity and headache activity measures, measures on quality of life and disability and non-headache measures. ${ }^{46}$ Headache frequency was not consistently used as primary outcome in included studies. However, the lower limit of the 95\% CI for a benefit on headache frequency effectively excludes any meaningful benefit on this outcome in the populations studied.

\section{Strengths and limitations}

Our analysis enabled us to quantitatively assess overall effectiveness of non-pharmacological self-management interventions versus usual care and to provide preliminary assessment of some intervention features associated with better outcomes in such self-management interventions. Our analyses specifically refer to the interventions meeting our inclusion criteria only; we note that the included intervention components are not comprehensive (eg, we a priori excluded biofeedback, physical exercise and interventions using apparatus) and our framework of self-management intervention components is not universally applicable.

A language bias cannot be ruled out as we only included trials in English. In addition, the initial screening stage was not double-coded. However, we have double-coded all potentially eligible studies by full-text screening and have checked reference lists of all included studies and related relevant publications to ensure we have not missed relevant studies.

Caution is needed when interpreting the analysis of intervention components. We are constrained by the descriptions of the interventions provided by the original authors, the distinctions between different intervention components may be unclear, and the amount of useable data is limited. Our categorisation framework relied on the original authors' description of the content of their interventions, and it is likely that there is overlap between categories. Nevertheless, we still identified some possible differences based on the described components; any misclassification of interventions would tend to reduce apparent effects. However, our analysis ignores any possible additive and/or synergistic effects of several components, which is important, as the combination of components is a relevant theoretical governing principle of the design of many trials. With the pragmatic approach used to explore components, any possible interplay between intervention components and delivery and population cannot be taken into account.

We included headache populations of interest regardless of the headache frequency or type. It would be preferable to consider each phenotypic group separately. However, the heterogeneity of the included populations and the descriptions means that any such approach would render any meaningful statistical analyses impossible. Nevertheless, we suggest that similarities between the different phenotypic groups included in these analyses are greater than their differences, meaning that our findings are directly applicable to the development of interventions to help people with migraine or tensiontype headaches to live better.

For the component analysis we only included comparisons with at least 10 studies per comparison (in line with previous research ${ }^{47}$ ). Thus there were many outcomes that could not be included in the analysis as data were too scarce, therefore assessing the effects of including particular delivery characteristics and components was not possible for several of the outcomes.

We did not have the data to assess the longer term effect of interventions. Where longer term follow-ups were reported in included studies, they included varied follow-up times, so we could not combine these in the present meta-analysis.

We used a pragmatic model of SMD meta-analyses and subgrouping to assess effectiveness as this approach was successfully used and established in a review with a similar research question in musculoskeletal pain. ${ }^{19}$ To interpret our results we used Cohen's $d$. We adopted this approach, as it is an approach recommended by the Cochrane Handbook..$^{20}$ However, the standardised mean difference cannot be translated into a clinical meaning and we urge caution when interpreting the results.

\section{CONCLUSIONS}

We found evidence that self-management interventions when compared with usual care for people living with migraine and tension-type headache improve many headache-related outcomes, but have no effect on headache frequency. We found some preliminarily evidence that delivery in groups, including CBT, educational and mindfulness components, appears to be associated with larger effects than those who did not. Although caution is required when interpreting the results, they provide some preliminarily evidence to guide research-based decisions about intervention content and delivery details of self-management interventions that aim to improve patients' capacity to manage their headaches.

Further research is required to confirm our results and to consider the optimal duration, and amount of contact hours and length of self-management interventions to help patients manage their headaches. Further methodological research is needed to explore the interactions between components and possible additive and/ or synergistic effects of components in self-management interventions. We would also urge authors of future trials to report data on intervention duration, attrition and contact hours (actual exposure) to the intervention, and include longer term follow-up in primary studies.

Acknowledgements Samantha Johnson (University of Warwick, academic support librarian) conducted the literature searches for this review.

Collaborators Achana, Felix; Warwick University, Division of Health Sciences Bright, Mary; University Hospitals Coventry and Warwickshire Caldwell, Fiona; Royal Holloway University of London Carnes, Dawn; Queen Mary University London Davies, Brendan; Royal Stoke University Hospital Eldridge, Sandra; Queen Mary University London Ellard, David; Warwick Clinical Trials Unit Evans, Simon; Migraine Action Griffiths, Frances; Warwick University, Division of Health Sciences Haywood, 
Kirstie; Warwick University, Division of Health Sciences Hee, Siew Wan; Warwick University, Division of Health Sciences Higgins, Helen; Warwick Clinical Trials Unit Matharu, Manjit; National Hospital for Neurology \& Neurosurgery Mistry, Hema; Warwick University, Division of Health Sciences Nichols, Vivien; Warwick Clinical Trials Unit Patel, Shilpa; Warwick Clinical Trials Unit Petrou, Stavros; Warwick University, Division of Health Sciences Pincus, Tamar; Royal Holloway University of London Potter, Rachel; Warwick Clinical Trials Unit Probyn, Katrin; Royal Holloway University of LondonSandhu, Harbinder; Warwick Clinical Trials Unit Taylor, Stephanie; Queen Mary University London Thomas, Wendy; The Migraine Trust Underwood, Martin; Warwick Clinical Trials Unit White, Kimberly; Warwick Clinical Trials Unit.

Contributors MU, TP and KP contributed to review concept and design; KP, FC and $\mathrm{HB}$ screened all search results and coded records for inclusion/exclusion; $\mathrm{KP}, \mathrm{HB}$, SP and HKS contributed to data extraction; DM conducted the statistical analysis $\mathrm{KP}, \mathrm{TP}$ and MU contributed to data interpretation. KP prepared the manuscript; all authors revised the manuscript critically for important intellectual content and approved the final version.

Funding This research was funded by the NIHR Programme Grants for Applied Research Programme (RP-PG-1212-20018). The views expressed in this publication are those of the author(s) and not necessarily those of the NHS, the NIHR or the Department of Health.

Competing interests MU reports grants from National Institute for Health Research, personal fees from National Institute for Health and Care Excellence, grants from Arthritis Research UK, personal fees from National Institute for Health Research, outside the submitted work; and chair of the guideline development group that produced the 2012 NICE headache guidelines. He has completed trials of manual therapy, group exercise and a cognitive behavioural approach as treatments for low back pain. He is a director and shareholder of Clinvivo Ltd.

Provenance and peer review Not commissioned; externally peer reviewed.

Data sharing statement The full data sets used and/or analysed are available from the corresponding author upon request.

Open Access This is an Open Access article distributed in accordance with the Creative Commons Attribution Non Commercial (CC BY-NC 4.0) license, which permits others to distribute, remix, adapt, build upon this work non-commercially, and license their derivative works on different terms, provided the original work is properly cited and the use is non-commercial. See: http://creativecommons.org/ licenses/by-nc/4.0/

(c) Article author(s) (or their employer(s) unless otherwise stated in the text of the article) 2017. All rights reserved. No commercial use is permitted unless otherwise expressly granted.

\section{REFERENCES}

1. Newman S, Steed L, Mulligan K. Self-management interventions for chronic illness. Lancet 2004;364:1523-37.

2. Smitherman TA, Houle DB, Penzien JC, et al. Headache: Hograefe, 2015.

3. Rains JC, Penzien DB, McCrory DC, et al. Behavioral headache treatment: history, review of the empirical literature, and methodological critique. Headache 2005;45(Suppl 2):S92-S109.

4. Andrasik F. Behavioral treatment of migraine: current status and future directions. Expert Rev Neurother 2004;4:403-13.

5. Biondi DM. Physical treatments for headache: a structured review. Headache 2005;45:738-46.

6. Baillie LE, Gabriele JM, Penzien DB. A systematic review of behavioral headache interventions with an aerobic exercise component. Headache 2014;54:40-53.

7. Ruth E, Goslin RNG, Douglas C, et al. Behavioral and physical treatments for Migraine Headache. Technical Reviews, No. 2.2 Rockville (MD): Agency for Health Care Policy and Research (US): Center for Clinical Health Policy Research, Duke University, 1999.

8 Linde K, Allais G, Brinkhaus B, et al. Acupuncture for the prevention of tension-type headache. Cochrane Database Syst Rev 2016;4:CD007587.

9. Linde K, Allais G, Brinkhaus B, et al. Acupuncture for the prevention of episodic migraine. Cochrane Database Syst Rev 2016;6:CD001218.

10. Harris P, Loveman E, Clegg A, et al. Systematic review of cognitive behavioural therapy for the management of headaches and migraines in adults. Br J Pain 2015;9:213-24.
11. Sullivan A, Cousins S, Ridsdale L. Psychological interventions for migraine: a systematic review. J Neurol 2016;263:2369-77.

12. (NICE) NloCE. Headaches: diagnosis and management of headaches in young people and adults. London, 2012.

13. Williams AC, Eccleston C, Morley S. Psychological therapies for the management of chronic pain (excluding headache) in adults. Cochrane Database Syst Rev 2012;11:CD007407.

14. Gibson PG, Powell H, Coughlan J, et al. Self-management education and regular practitioner review for adults with asthma. Cochrane Database Syst Rev 2003;1:CD001117.

15. Deakin T, McShane CE, Cade JE, et al. Group based training for self-management strategies in people with type 2 diabetes mellitus. Cochrane Database Syst Rev 2005;2:CD003417.

16. PROSPERO International prospective register of systematic reviews [Internet]. 2016. http://www.crd.york.ac.uk/PROSPERO/display_ record.asp?ID=CRD42016041291

17. Moher D, Liberati A, Tetzlaff J, et al. Preferred reporting items for systematic reviews and meta-analyses: the PRISMA statement. PLoS Med 2009;6:e1000097-676.

18. EPPI Reviewer 4. London: The Evidence for Policy and Practice Information and Co-ordinating Centre (EPPI-Centre), Social Science Research Unit at the Institute of Education, University of London.

19. Carnes D, Homer KE, Miles CL, et al. Effective delivery styles and content for self-management interventions for chronic musculoskeletal pain: a systematic literature review. Clin J Pain 2012;28:344-54.

20. Higgins Jpt GS. Cochrane Handbook for Systematic Reviews of Interventions: The Cochrane Collaboration, 2011. http://www. cochrane-handbook.org

21. Cosco TD, Doyle F, Ward M, et al. Latent structure of the Hospital Anxiety And Depression Scale: a 10-year systematic review. $J$ Psychosom Res 2012;72:180-4.

22. Stata v 14.4 College Station, Texas StataCorp LLC.

23. Cohen J. Statistical power analysis in the Behavioral Sciences. 2nd ed. Hillsdale (NJ): Lawrence Erlbaum Associates Inc., 1988.

24. Basler HD, Jakle C, Kroner-Herwig B. Cognitive-behavioral therapy for chronic headache at german pain centers. International Journal of Rehabilitation and Health 1996;2:235-52.

25. Bromberg J, Wood ME, Black RA, et al. A randomized trial of a web-based intervention to improve migraine self-management and coping. Headache 2012;52:244-61.

26. Cathcart S, Galatis N, Immink M, et al. Brief mindfulness-based therapy for chronic tension-type headache: a randomized controlled pilot study. Behav Cogn Psychother 2014;42:1-15.

27. Cousins SR, Ridsdale L, Goldstein LH, et al. A pilot study of cognitive behavioural therapy and relaxation. J Neurol 2015;262:2764-72.

28. D'Souza PJ, Lumley MA, Kraft CA, et al. Relaxation training and written emotional disclosure for tension or migraine headaches: a randomized, controlled trial. Ann Behav Med 2008;36:21-32.

29. Day MA, Thorn BE, Ward LC, et al. Mindfulness-based cognitive therapy for the treatment of headache pain: a pilot study. Clin J Pain 2014;30:152-61.

30. Devineni T, Blanchard EB. A randomized controlled trial of an internet-based treatment for chronic headache. Behav Res Ther 2005;43:277-92.

31. Mahmoudzadeh Zarandi F, Raiesifar A, Ebadi A. The effect of Orem's Self-Care Model on Quality of Life in Patients with Migraine: a Randomized Clinical Trial. Acta Med Iran 2016;54:159-64.

32. Martin PR, Reece J, Callan M, et al. Behavioral management of the triggers of recurrent headache: a randomized controlled trial. Behav Res Ther 2014:61:1-11.

33. Matchar DB, Harpole L, Samsa GP, et al. The headache management trial: a randomized study of coordinated care. Headache 2008:48:1294-310.

34. Mo'tamedi H, Rezaiemaram P, Tavallaie A. The effectiveness of a group-based acceptance and commitment additive therapy on rehabilitation of female outpatients with chronic headache: preliminary findings reducing 3 dimensions of headache impact. Headache 2012;52:1106-19.

35. Mosley HT, Grothues AC, Meeks WM. Treatment of Tension Headache in the elderly: a controlled evaluation of relaxation training and relaxation training combinded with cognitive-behavoural therapy. $J$ Clin Geropsychol 1995:175-88.

36. Omidi A, Zargar F. Effects of mindfulness-based stress reduction on perceived stress and psychological health in patients with tension headache. J Res Med Sci 2015;20:1058-63.

37. Sorbi MJ, Kleiboer AM, van Silfhout HG, et al. Medium-term effectiveness of online behavioral training in migraine selfmanagement: a randomized trial controlled over 10 months. Cephalalgia 2015;35:608-18. 
38. Ström L, Pettersson R, Andersson G. A controlled trial of self-help treatment of recurrent headache conducted via the Internet. $J$ Consult Clin Psychol 2000;68:722-7.

39. ter Kuile MM, Spinhoven P, Linssen AC, et al. Autogenic training and cognitive self-hypnosis for the treatment of recurrent headaches in three different subject groups. Pain 1994;58:331-40.

40. Kindelan-Calvo P, Gil-Martínez A, Paris-Alemany A, et al. Effectiveness of therapeutic patient education for adults with migraine. A systematic review and meta-analysis of randomized controlled trials. Pain Med 2014:15:1619-36.

41. Omidi A, Mohammadkhani P, Mohammadi A, et al. Comparing mindfulness based cognitive therapy and traditional cognitive behavior therapy with treatments as usual on reduction of major depressive disorder symptoms. Iran Red Crescent Med J 2013;15:142-6.

42. Segal ZV WM, Teasdale JD. Mindfulness-Based cognitive therapy for depression. New York: Guildford Press, 2002.
43. Pincus T, Holt N, Vogel S, et al. Cognitive and affective reassurance and patient outcomes in primary care: a systematic review. Pain 2013:154:2407-16.

44. Foster G, Taylor SJ, Eldridge SE, et al. Self-management education programmes by lay leaders for people with chronic conditions. Cochrane Database Syst Rev 2007;4.:CD005108.

45. Dindo LN. One-day behavioral intervention for depression and impairment in patients with comorbid depression and migraine. Headache 2014;54:1431-2.

46. Andrasik F, Lipchik GL, McCrory DC, et al. Outcome measurement in behavioral headache research: headache parameters and psychosocial outcomes. Headache 2005;45:429-37.

47. Miles CL, Pincus T, Carnes D, et al. Can we identify how programmes aimed at promoting self-management in musculoskeletal pain work and who benefits? A systematic review of sub-group analysis within RCTs. Eur J Pain 2011;15:1-11. 